\title{
The high-frequency QPOs in GRS 1915+105
}

\author{
T. Belloni ${ }^{1}$, M. Méndez ${ }^{2,3}$, and C. Sánchez-Fernández ${ }^{4}$ \\ 1 Osservatorio Astronomico di Brera, Via E. Bianchi 46, 23807 Merate (LC), Italy \\ 2 Astronomical Institute "A. Pannekoek" and Center for High-Energy Astrophysics, University of Amsterdam, \\ Kruislaan 403, 1098 SJ Amsterdam, The Netherlands \\ e-mail: mariano@astro.uva.nl \\ 3 Facultad de Ciencias Astronómicas y Geofísicas, Universidad Nacional de La Plata, Paseo del Bosque S/N, \\ 1900 La Plata, Argentina \\ e-mail: mmendez@fcaglp.unlp.edu.ar \\ 4 Laboratorio de Astrofísica Espacial y Física Fundamental (LAEFF-INTA), PO Box 50727, 28080 Madrid, Spain \\ e-mail: celia@laeff.esa.es
}

Received 5 January 2001 / Accepted 26 March 2001

\begin{abstract}
We analyzed two 1996 observations of the Galactic Microquasar GRS 1915+105 taken with the Proportional Counter Array on board the Rossi X-ray Timing Explorer. We focus on the properties of the highfrequency QPO as a function of the "dip" oscillations, a factor of 1000 slower. In one observation, 1996 May 5th, we find that the energy spectrum of the QPO at $\sim 65 \mathrm{~Hz}$ changes drastically between the high-intervals and the dips, hardening during the dips to the point that the QPO peak is no longer detected at low energies. In the second observation, 1996 May 14th, although it has similar overall characteristics, the $65 \mathrm{~Hz}$ QPO is not seen, but another broader QPO peak appears at $27 \mathrm{~Hz}$, only during the dips. This peak is too weak to obtain reliable information about its spectrum. Our results indicate that the presence/absence of high-frequency features in this enigmatic source are intimately linked to the slower oscillations and variations that happen on longer time scales.
\end{abstract}

Key words. accretion: accretion disks - black hole physics - stars: black hole candidates: oscillations X-rays: stars

\section{Introduction}

The X-ray source GRS 1915+105 was discovered in 1992 with WATCH (Castro-Tirado et al. 1992) as a bright transient (Castro-Tirado et al. 1994). Since then it has remained bright and active. It was the first galactic object to show superluminal expansion in radio observations (Mirabel \& Rodríguez 1994). It is considered a black-hole candidate because of its high X-ray luminosity and of its similarity with GRO J1655-40, for which a dynamical estimate of the mass of the compact object has been obtained (Bailyn et al. 1995).

A very large and valuable database of observations has been obtained with the Rossi X-ray Timing Explorer (RXTE). It is clear from RXTE data that the source shows a level of variability not seen in any other system (Greiner et al. 1996; Belloni et al. 1997a, 1997b; Chen et al. 1997; Muno et al. 1999). Belloni et al. (1997a, 1997b) interpreted this variability as the result of an instability that causes the innermost region of the accretion disk to disappear

Send offprint requests to: T. Belloni, e-mail: belloni@merate.mi.astro.it and be refilled again on a viscous time scale. These events are clearly associated with radio activity and jet ejection (Fender et al. 1997; Eikenberry et al. 1998; Mirabel et al. 1998; Fender \& Pooley 1998; Klein-Wolt et al., in preparation). Belloni et al. (2000) reduced the modes of variability into a number of separate classes, and concluded that all variations could be reduced to the alternation of three basic states, called A, B and C. In their interpretation, the major events are caused by $\mathrm{B}-\mathrm{C}$ transitions, with $\mathrm{C}$ being the state where the instability is at work and B the state where the full inner accretion disk is observable. The new state, state A, seems to be a separate third state where the accretion disk is fully observable, but softer than in state B.

The complexity of this source extends to the fast aperiodic variability. In the RXTE data, besides strong band-limited noise components, three different types of Quasi Periodic Oscillations (QPO) have been observed. Low-frequency QPOs at frequencies $\leq 0.1 \mathrm{~Hz}$ are observed (Morgan et al. 1997, hereafter MRG97), obviously associated with the long time scale variations caused by state changes (see Belloni et al. 2000). Intermediate-frequency 
QPOs, traditionally called $1-10 \mathrm{~Hz}$ QPOs are also observed. They are seen only during state $\mathrm{C}$ intervals, are strongly correlated with the source flux and energy spectrum, and show a complex phase-lag structure (MRG97; Markwardt et al. 1999; Reig et al. 2000). These 1-10 Hz QPOs show strong similarities with other QPOs observed in BHCs (see van der Klis 1995). In addition, a QPO at a rather constant frequency of $65-67 \mathrm{~Hz}$ has been observed in some observations (MRG97). Because its centroid frequency was seen to vary only by a couple of Hertz, this QPO is thought to be associated with a basic frequency of the system, such as the Keplerian frequency at the innermost stable orbit (MRG97), the Lense-Thirring precession at the same orbit (Cui et al. 1998), or a diskoseismic $g$ mode of the disk (Nowak et al. 1997). Other characteristics are its relatively high $Q$ value (defined as $\nu / \Delta \nu$ ) of $\sim 15$ (MRG97), its hard spectrum (MRG97) and its hard phase lags (Cui 1999). This feature has a fractional rms of only a few $\%$ and seems to be detected only during states A and $\mathrm{B}$, being therefore mutually exclusive with the $1-10 \mathrm{~Hz}$ QPO (see MRG97). Indeed, not all detections reported by MRG97 are very significant, and a full search for the presence of this QPO has not been yet published.

In this paper, we analyze two RXTE observations of GRS 1915+105 from 1996 May, where the $67 \mathrm{~Hz}$ QPO has been detected. In these observations strong oscillations with a time-scale of $\sim 10 \mathrm{~s}$ are observed (see MRG97), corresponding to oscillations between state $\mathrm{A}$ and state B (see Belloni et al. 2000). We explore the relation between the high-frequency QPO and these low-frequency state changes.

\section{Data analysis}

\subsection{Selection of observations}

MRG97 report the detection of the $67 \mathrm{~Hz}$ QPO in 6 observations, all made in the first half of 1996. Some of the detections have a very low significance. All these observations, with the exception of the first (Apr 6th), correspond to class $\gamma$ in the classification by Belloni et al. (2000). This class is indeed characterized by oscillations on a $\sim 10$ s time scale (see Fig. 9 in MRG97), oscillations that are accompanied by spectral changes (softening during the narrow dips). In order to analyze in detail whether the high-frequency QPO shows variations within one observation, a strong QPO detection is needed. Moreover, in order to study its possible connection with the lowfrequency oscillation ( $100 \mathrm{mHz}$, see MRG97), it was necessary to obtain a good template for the latter. For this purpose, the low-frequency oscillation needs to be rather regular. Only one observation meets both requirements, that of May 5th, which was also analyzed by Cui (1999), who concentrated on the phase lags of the different oscillations. In addition, we analyzed another of those observations, May 14th, where the $67 \mathrm{~Hz}$ QPO over the whole observation has a low significance ( $2 \sigma$ in MRG97), but the low-frequency oscillation is rather regular.

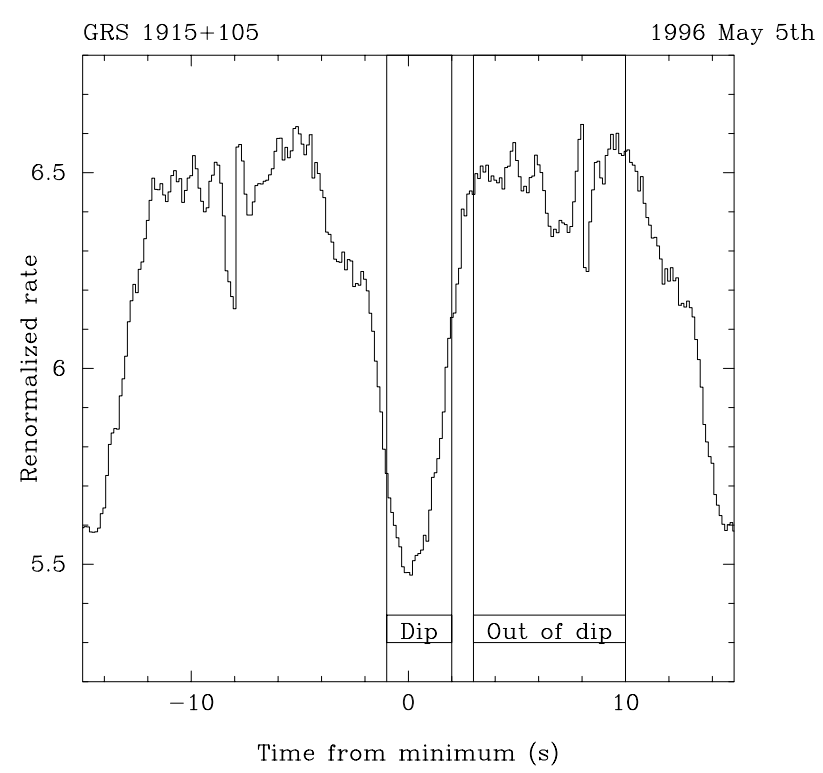

Fig. 1. Folded light curve for the May 5th observation obtained by aligning the minima (see text). The marked bands are those used for the production of the "dip" and "out of dip" PDSs.

\subsection{The May 5th observation}

For each of the two observations, in order to identify the times of dips, we followed a procedure similar to that adopted by MRG97. First we produced a light curve in the $2-13 \mathrm{keV}$ band, with a bin size of $0.125 \mathrm{~s}$. Then we ran a sliding window with a $16 \mathrm{~s}$ width on the light curve. For each window, we fitted to the data a model consisting of a constant plus a negative Gaussian profile centered on the middle of the window. For each fit, free parameters were therefore the constant level, and width and normalization of the Gaussian. As the next step, we identified the times of the dips as local minima of the best fit amplitude of the Gaussian amplitude in a 5-second time window, with the additional constraint that the amplitude had to be less than $-1000 \mathrm{cts} / \mathrm{s}$. Visual inspection of the light curve showed that this was a reasonable way to identify the minima, which are relatively easy to see by eye. A few spurious minima were deleted by hand, the level of $-1000 \mathrm{cts} / \mathrm{s}$ was chosen to be conservative. With the minima, we constructed a template light curve by summing the data centered at the times of the minima. The resulting template for May 5 th can be seen in Fig. 1. The spikes at $-8 \mathrm{~s}$ and $+8 \mathrm{~s}$ are spurious, corresponding to the limits of the window used for the fits. The curve is similar to that in MRG97, although their procedure was slightly different: the oscillation is highly non-sinusoidal, being constituted by a sharp dip every $\sim 15 \mathrm{~s}$

From our template, we identified two intervals: a $3 \mathrm{~s}$ interval around the minimum, corresponding to the "dip", and a $7 \mathrm{~s}$ interval where the flux is at its maximum and rather constant level (see Fig. 1). The $3 \mathrm{~s}$ dip interval is not symmetric around the minimum since from the procedure for the extraction of PDSs (see below), we were limited to intervals $1 \mathrm{~s}$ long. A selection with the dip 
Table 1. Best fit parameters for the PDSs showed in Figs. 2 and 4 . Upper limits are at 95\% confidence. (FIX) indicates that a parameter has been fixed to the quoted value (see text).

\begin{tabular}{lcccccc}
\hline Interval & $\nu_{0}(\mathrm{~Hz})$ & $\Delta(\mathrm{Hz})$ & $\%$ rms & $\chi^{2}$ (d.o.f.) & $H R_{1}$ & $H R_{2}$ \\
\hline \multicolumn{7}{c}{1996 May 5 th $(2-13 \mathrm{keV})$} \\
\hline Out of dip & $65.01 \pm 0.17$ & $4.69 \pm 0.45$ & $4.32 \pm 0.20$ & $51(45)$ & $1.2248 \pm 0.0003$ & $0.1245 \pm 0.0001$ \\
Dip & $65.01($ FIX) & $4.69($ FIX) & $<2.49(95 \%)$ & $53(47)$ & $1.1773 \pm 0.0005$ & $0.1248 \pm 0.0002$ \\
\hline \multicolumn{7}{c}{1996 May 5 th $(13-30 \mathrm{keV})$} \\
\hline Out of dip & $64.87 \pm 0.27$ & $4.41 \pm 0.84$ & $5.52 \pm 0.42$ & $37(45)$ \\
Dip & $64.81 \pm 0.24$ & $2.74 \pm 0.87$ & $5.56 \pm 0.56$ & $37(45)$ \\
\hline \multicolumn{7}{c}{1996 May 14 th $(2-13 \mathrm{keV})$} \\
\hline Dip & $27.09 \pm 0.36$ & $9.08 \pm 1.15$ & $2.24 \pm 0.15$ & $94(84)$ & $1.1432 \pm 0.0005$ & $0.1459 \pm 0.0002$ \\
Out of dip & $27.09($ FIX) & $9.08($ FIX) & $<0.85(95 \%)$ & $140(86)$ & $1.0626 \pm 0.0004$ & $0.1470 \pm 0.0002$ \\
\hline
\end{tabular}

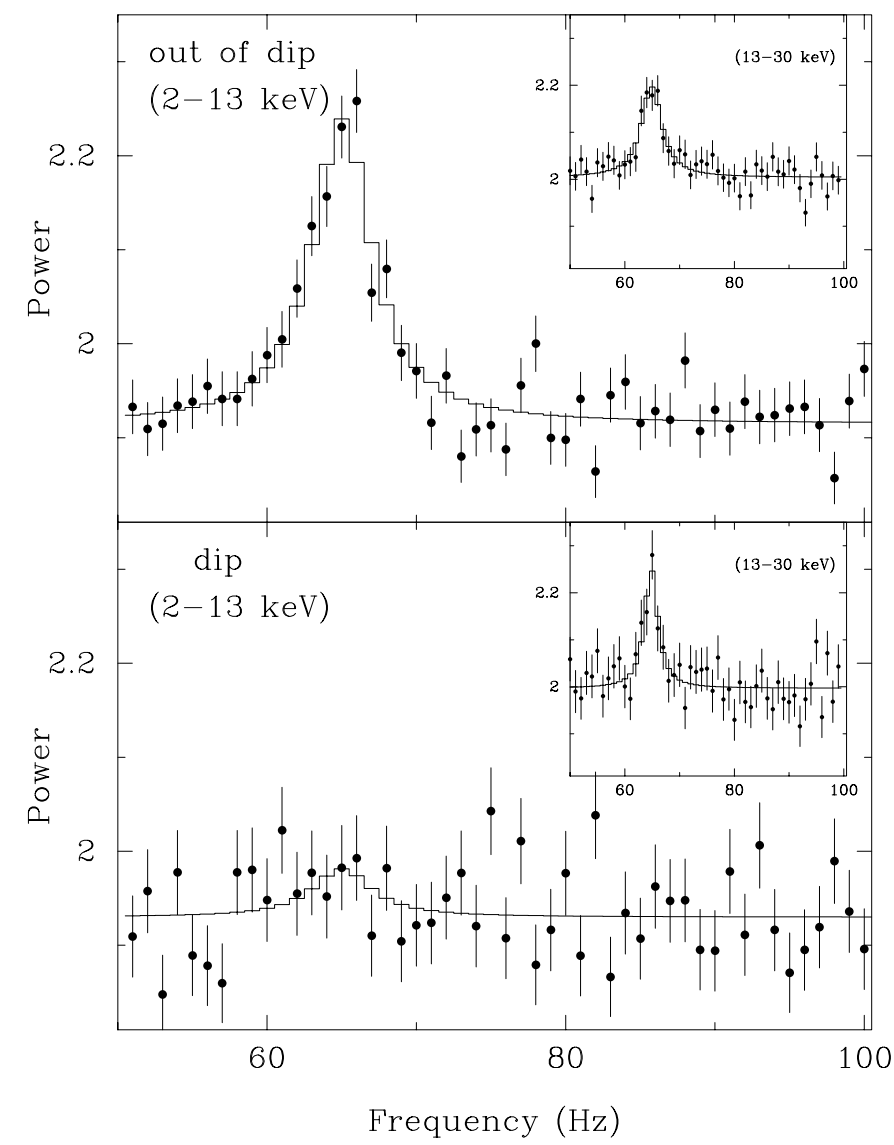

Fig. 2. High-frequency part of the PDSs obtained accumulating "out of dip" (upper panel) and "dip" (lower panel) intervals $(2-13 \mathrm{keV})$. The lines indicate the best fit models (see text). In the smaller panels, the corresponding PDSs for the $13-30 \mathrm{keV}$ band are shown.

interval shifted backwards by $1 \mathrm{~s}$ gave compatible results. We then divided the same $2-13 \mathrm{keV}$ light curve in intervals 1 second long and accumulated a Power Density Spectrum (PDS) for each of the intervals, at the highest resolution available (2 ms), corresponding to a Nyquist frequency of
$256 \mathrm{~Hz}$ ). The PDSs were normalized according to Leahy et al. (1983). Finally, we summed all the PDSs corresponding to the "dip" and "out of dip" times described above to obtain the average PDS for each of the two intervals. The results can be seen in Fig. 2. It is evident that the QPO at $\sim 65 \mathrm{~Hz}$ is present only in the high-flux PDS. We fitted the "out of dip" PDS in Fig. 2 with a model consisting of a Lorentzian (with centroid frequency $\nu_{0}$ and FWHM $\Delta$ ) for the QPO peak and a constant to take into account the contribution by the Poissonian noise. The best fit value for the QPO power has been converted to fractional $\mathrm{rms}$ by taking into account the net source rate, after subtraction of the average background rate estimated with the FTOOL pcabackest. The best fit parameters can be seen in Table 1. The QPO peak at $65 \mathrm{~Hz}$ has a fractional $\mathrm{rms}$ amplitude of $4.32 \pm 0.20 \%$ (significant at a $11.3 \sigma$ level) in the $2-13 \mathrm{keV}$ energy range. For fitting the "dip" PDS, we fixed centroid and width of the Lorentzian to the "out of dip" values and obtained a $95 \%$ upper limit on the fractional rms of the QPO of $2.49 \%$, significantly lower than the detection outside the dips.

Since in MRG97 the fractional rms of the $65 \mathrm{~Hz}$ QPO increases with increasing energy, we produced power density spectra also for a higher energy band (13-30 keV). In this band, the $65 \mathrm{~Hz}$ QPO is detected both in the dips and outside $(5.0 \sigma$ and $6.5 \sigma$ respectively, see small panels in Fig. 2), with a centroid frequency compatible with that of the softer energy band, with a fractional $\mathrm{rms}$ of $\sim 5.5 \%$, similar to that reported by MRG97 (see Table 1). The only difference here is that the QPO peak is narrower during the dips $(2.74 \pm 0.87 \mathrm{~Hz})$ than outside $(4.41 \pm 0.84 \mathrm{~Hz})$.

\subsection{The May 14th observation}

We repeated the same procedure for the May 14th observation. Our hope was to be able to extract a more significant detection for the $65 \mathrm{~Hz}$ QPO by de-selecting the dip intervals. As already shown by MRG97, the low-frequency oscillation in this observation is faster $(\sim 9 \mathrm{~s})$, and its shape 


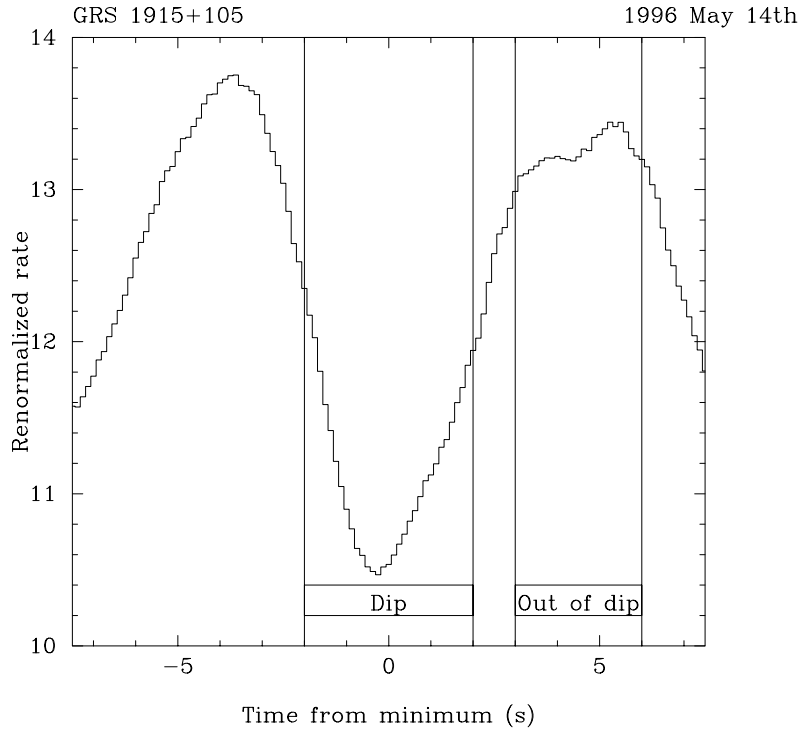

Fig. 3. Folded light curve for the May 14th observation obtained by aligning the minima (see text). The marked bands are those used for the production of the "dip" and "out of dip" PDSs.

is roughly sinusoidal, therefore different from the sharp dips shown before. This can be seen in the template shown in Fig. 3. Nevertheless, we selected a 4 s stretch around the minimum, which we call for consistency "dip", and a $3 \mathrm{~s}$ stretch around the maximum as "out of dip" (see Fig. 3). The resulting averaged PDSs $(2-13 \mathrm{keV})$ can be seen in Fig. 4. No significant peak is present around $65 \mathrm{~Hz}$ in either spectrum, but a broad peak around $27 \mathrm{~Hz}$ is visible in the "dip" spectrum. This peak is easily seen also in the total PDS shown by MRG97.

Again, we fitted the PDSs, adding to the model described above a power law to describe the low-frequency excess, which we could not simply window out as in the previous case. The parameters are shown in Table 1. In the "dip", the QPO peak at $27 \mathrm{~Hz}$ has a fractional rms amplitude of $2.24 \pm 0.15 \%$ (significant at the $7.5 \sigma$ level). Outside the dips, fixing the QPO parameters to the "dip" ones, we obtained a $95 \%$ upper limit on the fractional $\mathrm{rms}$ of a $27 \mathrm{~Hz}$ peak of $0.85 \%$.

As in the previous case, we repeated the analysis in the 13-30 keV band, but found no QPO at the same frequency. The derived upper limits are not very stringent and completely compatible with the low-frequency detection $(2.9 \%$ and $4.5 \%$ for the out-of-dip and dip respectively).

\subsection{Spectral variations during the dips}

In order to check whether the observed dips are indeed transitions from state B to state A of Belloni et al. (2000), since these two observations were not included in their sample, we accumulated X-ray colors for the dip and out of dip intervals. We chose the same X-ray bands and the same definitions of colors as in Belloni et al. (2000). Colors are defined as $H R_{1}=\mathrm{B} / \mathrm{A}$ and $H R_{2}=\mathrm{C} / \mathrm{A}$, where $\mathrm{A}, \mathrm{B}$

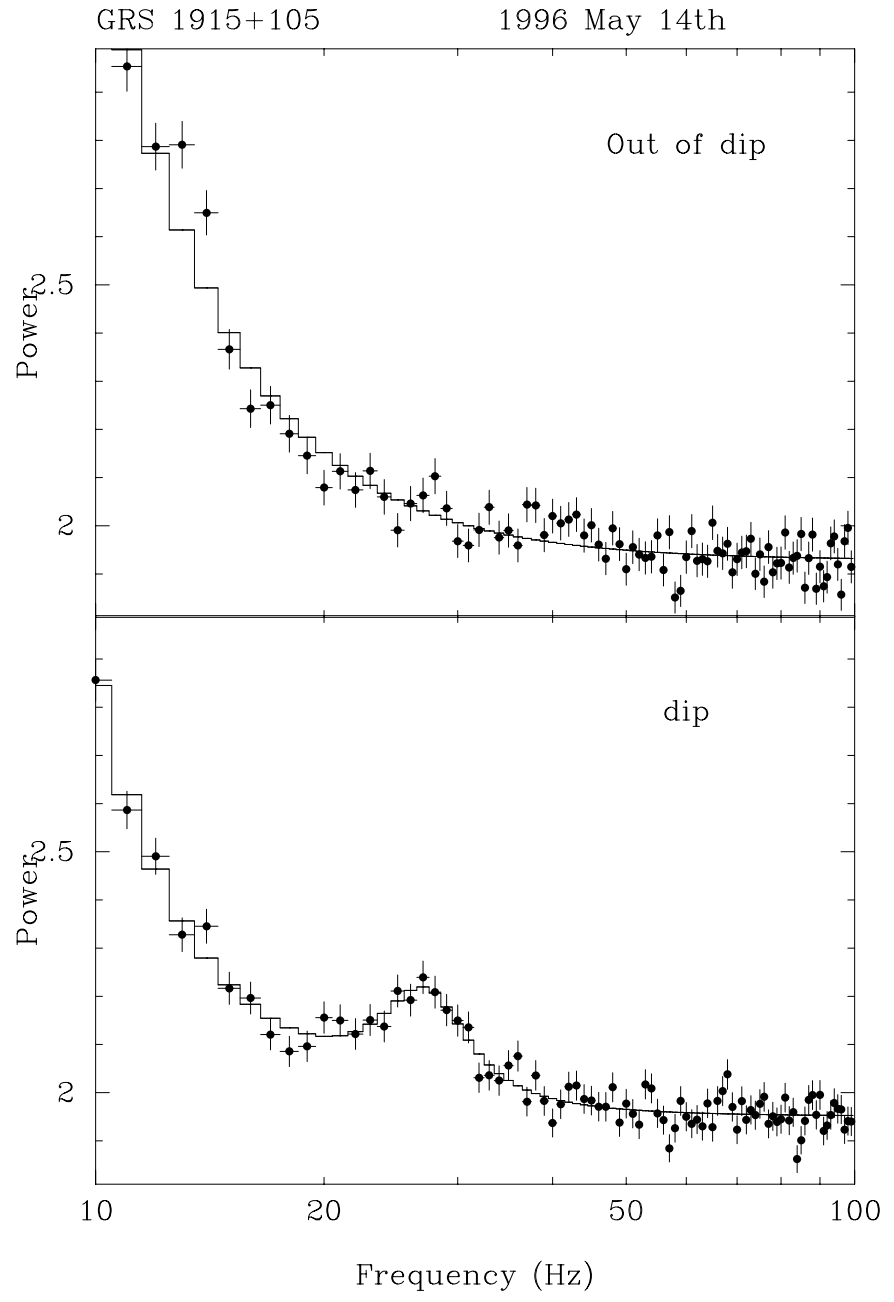

Fig. 4. High-frequency part of the PDSs obtained accumulating "out of dip" (upper panel) and "dip" (lower panel) intervals $(2-13 \mathrm{keV})$. The lines indicate the best fit models (see text).

and $\mathrm{C}$ are the net source counts in the channel bands $0-13$ (2-5 keV), 14-35 (5-13 keV), and 36-255 (13-60 keV) respectively. For each observation, all the dip and out-of-dip data were summed to compute the colors. The result is the same for both observations: the soft color $H R_{1}$ decreases significantly during the dips, while the hard color does not change within the errors for May 5th, and decreases very slightly on May 14th. This behaviour is consistent with A-B transitions (see Belloni et al. 2000).

To investigate more thoroughly the spectral variations of the May 5th observations, we extracted energy spectra from the PCA data, using the high time resolution data modes (binned and event modes). The spectral resolution of these modes for this observation is rather low, featuring only four independent channels below $13.5 \mathrm{keV}$, but it is important to have an idea of the energy spectrum and to calculate the relative contribution of the disk and power-law component. We extracted the spectra and the PCA response matrices using FTOOLS V5.0.1. Before extracting the spectra for our aims, we tested our extraction procedure by accumulating a $96 \mathrm{~s}$ spectrum both from high time resolution data and from standard data, which 
have full energy resolution: we obtained compatible results. We then accumulated two spectra, corresponding to the dip and out-of-dip intervals used for the production of the PDSs (see Sect. 2.2). We fitted both spectra (excluding the first channel and the channels above $25 \mathrm{keV}$ because of calibration uncertainties at low and high energies, effectively restricting ourselves to the $5-25 \mathrm{keV}$ interval), with a model consisting of a disk-blackbody plus a power law (with an artificial low-energy break at $4 \mathrm{keV}$ in order to avoid a power-law excess at low energies). The interstellar absorption was fixed to $6 \times 10^{22} \mathrm{~cm}^{-2}$ (see Belloni et al. 2000). A $1 \%$ systematic error was added to all channels to account for residual calibration uncertainties. The fits proved rather problematic: no good fit in the statistical sense could be obtained, with reduced $\chi^{2}$ values of 2.8 and 4.0 ( 6 d.o.f.) for the out-of-dip and dip spectrum respectively. At this stage, we are not able to provide a robust estimate of the parameter changes that give rise to the difference in the observed colors. Overall, as expected (see Belloni et al. 2000), the disk has a rather high temperature in both spectra $(\sim 2 \mathrm{keV})$ and a small inner radius $(\sim 20 \mathrm{~km})$, while the power law is steep $(\Gamma \sim 3.5)$. However, given the relatively small changes of the spectral parameters between the two spectra (the only significant change is the inner radius of the disk component, larger in the outof-dip spectrum, we can estimate roughly the percentage of detected photons coming from the two components. In the out-of-dip spectrum, the power law contributes $49 \%$ of the total observed counts in the low-energy band, and about the same percentage in the high-energy band. In the dip spectrum, these percentages are $52 \%$ and $50 \%$ respectively. From the uncertainties in the spectral parameters, we can estimate an error on these percentages of $\pm 3 \%$.

\section{Discussion}

The analysis presented in the previous section shows that there is a relation between the low-frequency oscillation and the presence of the high-frequency QPO, on a time scale a factor of $\sim 1000$ shorter. However, this relation is complex and difficult to interpret. The energy spectrum of black hole candidates in general and of GRS 1915+105 in particular can be decomposed into two separate components: a thermal component thought to originate from an optically thick accretion disk and a high-energy component associated with an optically thin flow (see Tanaka \& Lewin 1995; Belloni et al. 1997a, 1997b). The energy dependence of the fractional rms of the $65 \mathrm{~Hz}$ QPO analyzed in this work is rather hard, indicating that it must be associated with the optically thin component (MRG97). In the following, we will make this assumption.

Let's examine the two different PDSs of the May 5th observation.

- Out-of-dip: in this case, the fractional rms of the detected $65 \mathrm{~Hz}$ QPO is $4.3 \%$ in the soft energy band and $5.5 \%$ in the hard energy band. (see Sect. 2.2). These figures have been calculated by assuming the QPO is associated with the total flux from the source. If we make the assumption that the QPO comes only from the power-law component, using the fraction of counts from the power-law component from Sect. 2.4, the fractional $\mathrm{rms}$ in the soft energy band increases to $8.8 \%$, that in the hard energy band to $11.2 \%$;

- Dip: here the QPO has an upper limit of $2.5 \%$ in the soft band, and a detection of $5.5 \%$ in the hard band. Using the figures from Sect. 2.4, under the same assumption that the QPO is associated only with the power-law spectral component, we can convert these numbers to $4.8 \%$ and $11 \%$ respectively.

From this exercise, we can conclude a few things. The first is that the increase in the fractional rms of the QPO reported by MRG97, associated with the out-of-dip intervals, could be entirely due to the fact that the QPO is associated with the hard power-law component and at low energies is diluted by the disk component. The second is that we can exclude that the non-detection of the QPO in the soft band during the dips is due to a relative increase of the contribution of a non-variable disk component. This because after correcting for the power-law fraction (see above), the soft-band detection in the out-of-dip data is $8.8 \pm 0.7 \%$ and the $95 \%$ upper limit in the dip data is $4.8 \%$. An alternative way to estimate this effect is to calculate by how much the power-law fraction would have to decrease in the dip spectrum with respect to the out-of-dip spectrum in order to make the detection in the one compatible with the upper limit in the other. The resulting number is 0.58 , while within the errors the two fractions remain the same. Notice that also the amplitude of the low-frequency oscillation increases with energy (Tomsick \& Kaaret 2001), as do most of the QPOs observed in black hole candidates (see van der Klis 1995).

Unfortunately, a more detailed analysis is not possible due to the lack of energy resolution in the high time resolution energy spectra. However, our results show that the presence of the $65 \mathrm{~Hz}$ QPO, which has been reported only for a limited number of observations, is indeed limited to a very specific state of the source, which we identify with state B of Belloni et al. (2000). The periodic fast excursions to state A (the dips) are already enough to produce sharp changes in the properties of the QPO, whose spectrum must change in order not to have it detected in the soft energy band. A thorough analysis of a large number of PCA observations of GRS $1915+105$ is needed, but is clearly beyond the scope of this work.

Finally, the PDSs of May 14th show that we are far from having a complete picture of the timing properties of GRS 1915+105: here the $65 \mathrm{~Hz}$ does not seem to be significantly present in either the out-of-dip or the dip PDSs, but another QPO at $27 \mathrm{~Hz}$ appears, only during the dips. Not much more can be said about this, in the lack of additional datasets showing oscillations at these and possibly other frequencies. Of course, given the peculiar property of GRS $1915+105$ to change its visible inner disk radius over short time scales, none of the models proposed for 
the generation of a high-frequency QPO (see e.g. MRG97; Nowak et al. 1997; Cui et al. 1998) are ruled out by these results.

Acknowledgements. We thank S. Campana for useful discussions. TB thanks the Cariplo foundation for financial support. This work was supported by The Netherlands Research School for Astronomy (NOVA), The Netherlands Organization for Scientific Research (NWO) under contract number 614-51-002 and the NWO Spinoza grant 08-0 to E. P. J. van den Heuvel. $\mathrm{MM}$ is a fellow of the Consejo Nacional de Investigaciones Científicas y Técnicas de la República Argentina. CSF was partially supported by a "Rafael Calvo Rodes" fellowship from Instituto Nacional de Tecnica Aeroespacial, Spain. This research has made use of the data obtained through the High Energy Astrophysics Science Data Archive Research Center Online Service, provided by the NASA/Goddard Space Flight Center.

\section{References}

Bailyn, C. D., Orosz, J. A., McClintock, J. E., \& Remillard, R. A. 1995, Nature, 378, 157

Belloni, T., Méndez, M., King, A. R., van der Klis, M., \& van Paradijs, J. 1997a, ApJ, 479, L145

Belloni, T., Méndez, M., King, A. R., van der Klis, M., \& van Paradijs, J. 1997b, ApJ, 488, L109

Belloni, T., Klein-Wolt, M., Méndez, M., van der Klis, M., \& van Paradijs, J. 2000, A\&A, 355, 271

Castro-Tirado, A. J., Brandt, S., Lund, S. 1992, IAU Circ., 5590
Castro-Tirado, A. J., Brandt, S., Lund, S., et al. 1994, ApJS, 92, 469

Chen, X., Swank, J. H., \& Taam, R. E. 1997, ApJ, 477, L41

Cui, W., Zhang, S. N., \& Chen, W. 1998, ApJ, 492, L53

Cui, W. 1999, ApJ, 542, L59

Eikenberry, S. S., Matthews, K., Morgan, E. H., Remillard, R. A., \& Nelson, R. W. 1998, ApJ, 494, L61

Fender, R. P., Pooley, G. G., Brocksopp, C., \& Newell, S. J. 1997, MNRAS, 290, L65

Fender, R. P., \& Pooley, G. G. 1998, MNRAS, 300, 573

Greiner, J., Morgan, E. H., \& Remillard, R. A. 1996, ApJ, 473, L107

Leahy, D. A., Darbro, W., Elsner, R. F., et al. 1983, ApJ, 266, 160

Markwardt, C. B., Swank, J. H., \& Taam, R. E. 1999, ApJ, 513, L37

Mirabel, I. F., \& odríguez, L. F. 1994, Nature, 371, 46

Mirabel, I. F., Dhawan, V., Chaty, S., et al. 1998, A\&A, 330, L9

Morgan, E. H., Remillard, R. A., \& Greiner, J. 1997, ApJ, 482, 993 (MRG97)

Muno, M. P., Morgan, E. H., \& Remillard, R. A. 1999, ApJ, 527,321

Nowak, M. A., Wagoner, R. V., Begelman, M. C., \& Lehr, D. E. 1997, ApJ, 477, L91

Reig, P, Belloni, T., van der Klis, M., et al. 2000, ApJ, 541, 883

Tomsick, J. A., \& Kaaret, P. 2001, ApJ, 548, 401

van der Klis, M. 1995, in X-ray binaries, ed. W. H. G. Lewin, J. Van Paradijs, \& E. P. J. Van den Heuvel (Cambridge Univ. Press., Cambridge), 252 